Research Paper

\title{
Inhibition of Breast Tumor Cell Growth by Ectopic Expression of p16/INK4A Via Combined Eifects of Cell Cycle Arrest, Senescence and Apoptotic Induc- tion, and Angiogenesis Inhibition
}

\author{
Yi Lu1,2,3凶, Xiongwen Zhang ${ }^{2,4}$, and Jun Zhang1,3 \\ 1. Department of Pathology, University of Tennessee Health Science Center, Memphis, TN, USA \\ 2. Department of Urology, University of Tennessee Health Science Center, Memphis, TN, USA \\ 3. Department of Medicine, University of Tennessee Health Science Center, Memphis, TN, USA \\ 4. Division of Anticancer Pharmacology, Shanghai Institute of Materia Medica, Shanghai, PR China
}

Corresponding author: Yi Lu, Ph.D. Department of Pathology and Laboratory Medicine, University of Tennessee Health Science Center, Cancer Research Building, Room 218, 19 South Manassas Street, Memphis, TN 38163, USA. Tel: (901) 448-5436; Fax: (901) 448-5496; e-mail: ylu@uthsc.edu

(c) Ivyspring International Publisher. This is an open-access article distributed under the terms of the Creative Commons License (http://creativecommons.org/ licenses/by-nc-nd/3.0/). Reproduction is permitted for personal, noncommercial use, provided that the article is in whole, unmodified, and properly cited.

Received: 2012.01.03; Accepted: 2012.02.23; Published: 2012.08.01

\begin{abstract}
p 16-mediated inhibition of cancer cell proliferation and tumor suppression have been studied before,; the common consensus is that p16's cell-cycle arrest function plays a primary role in these actions, with some additional apoptotic induction by p 16 . However, other effects of p 16 that may potentially contribute to p 16-mediated anti-tumor ability have not been well studied. The emerging data including ours indicated that $\mathrm{p} / 6$ contributes its anti-cancer ability by inducing tumor cells to senescence. Moreover, we showed that pl 6 inhibits breast cancer cell growth by inhibiting the VEGF signaling pathway and angiogenesis. In this study, we used adenoviral-mediated pl6 expression (AdRSVp/6) and breast cancer cell line MDA-MB-23 I as the model to simultaneously analyze all these p16's anti-tumor functions. We demonstrated that adenoviral-mediated p 16 expression exhibited multiple anti-tumor functions by simultaneously suppressing in vitro growth and in vivo angiogenesis of breast cancer cells, blocking cell division, as well as inducing senescence and apoptosis. The in vivo study implies that pl6's effect on anti-angiogenesis may play a more significant role than its anti-cell proliferation in the overall suppression of tumor growth. These results suggest, for the first time, that AdRSV I 6-mediated tumor suppression results from a combination of pl6's multiple anti-tumor functions including p/6's well-known anti-proliferation/cell division function, apoptotic and senescence induction function, and its lesser-known/under-investigated anti-angiogenesis function. These combined results strongly indicate that $\mathrm{p} / 6$ gene therapy has a multi-module platform with different anti-tumor functions; therefore, this study justifies and promotes the viral-mediated pl6 gene therapy as a promising and powerful treatment approach for cancer patients due to pl6's multiple anti-tumor functions.
\end{abstract}

Key words: adenovirus, angiogenesis, apoptosis, breast cancer, gene therapy, p16, senescence

\section{Introduction}

The tumor suppressor gene p16 (also called MTS1, CDKN2 and INK4A) is a cyclin-dependent kinase (CDK) inhibitor and a negative cell cycle reg- ulator (1). The inactivation of p16 appears to be a common event in many cancers (2-9). Conversely, overexpression of VEGF, the primary angiogenic fac- 
tor that plays a pivotal role in tumor angiogenesis, is strongly associated with tumor malignancy (10). VEGF was shown in breast tumors to be at levels 7 -fold higher than in normal adjacent tissue (11). The expression of VEGF markedly contributes to tumor-associated neovascularization and is correlated with malignant transformation of breast cancer (BCa) and poor prognosis (12-16). Our previous study demonstrated that introduction of exogenous p16 via adenoviral gene transfer downregulated VEGF expression and inhibited angiogenesis in human BCa cells (17). These results imply that p16's anti-VEGF/anti-angiogenesis function may contribute to suppression of tumor growth.

Likewise, several other functions of p16, including induction of apoptosis and senescence, have also been studied and correlated to p16's anti-tumor/anti-proliferation effects. p16 is able to induce apoptosis of tumor cells: Adenoviral-mediated expression of p16 (Adp16) inhibits ovarian cancer cell growth and causes apoptosis (18). The combined overexpression of p16 and p53, but not p53 alone, induces efficient apoptotic death in a majority of tumors (19). Accumulated evidences have shown that senescence induction is linked to the p16's tumor inhibition. Malignant glioma cells showed increased growth arrest and induction of senescence after induction of p16 gene (21). A similar phenotype has also been described in glioma cells following adenoviral-mediated p16 gene transfer (22). Consistent with these studies, we previously demonstrated that senescence was induced in prostate cancer cells either by transduction of Adp16 or by treatment with 5-aza-2'-deoxycytidine, an agent that demethylates the p16 promoter $(23,24)$. While these emerging data among various cancer cells may suggest a link between certain individual p16's function and its potential unique anti-tumor ability through that particular function/pathway, the lack of a systemic and simultaneous analysis for all these p16 features (i.e., effects on tumor angiogenesis, proliferation, apoptosis and senescence) in one cancer cell line hinders the dissection of the role of each p16's individual function on its overall anti-tumor effect.

In this study, we individually examined each of p16's multiple functions on anti-proliferation, anti-VEGF/angiogenesis, apoptosis and senescence in the BCa cells in order to determine whether p16's overall inhibition of BCa cell growth and tumor suppression results from a combined effect from these individual functions. These results may justify and promote the viral-mediated p16 gene therapy as a promising treatment approach for cancer patients due to p16's multiple anti-tumor functions at different biological aspects.

\section{Materials and methods}

\section{Cell lines and tissue culture conditions}

The human BCa cell line MDA-MB-231 and MCF-7 cells were purchased from the American Type Culture Collection (ATCC, Manassas, VA). MDA-MB-231 cells were grown in RPMI-1640 medium (Cellgro, Herndon, VA) with 10\% fetal bovine serum (FBS, Hyclone Laboratories, Logan, Utah) and MCF-7 were grown in McCoy's $5 \alpha$ medium (Cellgro) with 10\% FBS. JygMC(A) (a generous gift from Dr. H. Azuma, Osaka Medical College, Osaka, Japan. Ref 20) and 293 cells (ATCC) were grown in Dulbecco's modified Eagle medium (Cellgro) with 10\% FBS. All cell lines were grown in medium containing 100 units $/ \mathrm{ml}$ penicillin, plus $100 \mathrm{mg} / \mathrm{ml}$ streptomycin at $37^{\circ} \mathrm{C}$ in $5 \% \mathrm{CO}_{2}$.

\section{Generation of recombinant adenoviral vectors}

The construction of AdRSVp16, a replication-defective, recombinant adenoviral vector expressing a human wild-type p16 cDNA gene under the control of a Rous sarcoma virus (RSV) promoter, was previously described (24). The construction of the control virus, AdRSVlacZ, in which a bacterial B-galactosidase gene (lacZ) was under the control of the RSV promoter, was also described previously (26).

\section{Adenoviral vector preparation, titration and transduction.}

Individual clones of adenoviral vector were propagated in 293 cells. The culture medium of the 293 cells showing the complete cytopathic effect was collected, and adenoviral vectors were purified by BD Adeno- $X$ Virus Purification Kits (BD Biosciences, Palo Alto, CA) according to the manufacturer's instructions. The viral titration and transduction were performed by standard procedures as previously described (27).

\section{Growth inhibition assay}

Cells were plated at a density of $2 \times 10^{4}$ cells per well in 6-well plates. After incubation for $24 \mathrm{~h}$, cells were transduced with AdRSVp16 or control virus at a different multiplicity of infection (moi) as indicated. The cell numbers were determined using a Coulter cell counter (Coulter, Hialeah, FL) at indicated days post-transduction. The results represent the data from at least two independent experiments, each performed in duplicate. The student t-test was used throughout this study for the statistical analysis. 


\section{Flow cytometry to detect cell cycle distribu- tion}

Cells were arrested at $\mathrm{G}_{0} / \mathrm{G}_{1}$ phase by serum starvation by treating cells with $0.5 \% \mathrm{FBS}$ at $37^{\circ} \mathrm{C}$ for $48 \mathrm{hr}$ (28). After washing the cells, the synchronized cells were replated in fresh medium containing $10 \% \mathrm{FBS}$ to reentering the cell cycle at $37^{\circ} \mathrm{C}$ for $16 \mathrm{~h}$, and the cells were then untreated or treated with viral transduction. Cellular DNA contents that represented the cell cycle phase $\left(\mathrm{G}_{0} / \mathrm{G}_{1}, \mathrm{~S}\right.$, or $\left.\mathrm{G}_{2} / \mathrm{M}\right)$ were detected by flow cytometry via determination of propidium iodide (29). After trypsinization, cells were washed with PBS and cell pellets were fixed in $70 \%$ ethanol at $4^{0} \mathrm{C}$ overnight. After being washed twice with PBS, the cells were stained with $50 \mathrm{ug} / \mathrm{ml}$ propidium iodide (Sigma, St. Louis, MO), $10 \mathrm{ug} / \mathrm{ml}$ RNase A, 0.5\% (v/v) Triton X-100 and $0.1 \%(\mathrm{w} / \mathrm{v})$ sodium citrate for $30 \mathrm{~min}$ at room temperature in the dark before fluorescent flow cytometry. The similar approach was used to detect the apoptotic cell population by measuring the pre-G1 phase (apoptotic cell population) of the cell cycle.

\section{Northern blot}

Cells were extracted, and total RNA was isolated using a RNeasy kit (Qiagen, Santa Clarita, CA). Total RNA was loaded on a $1.2 \%$ polyacrylamide gel and processed to electrophoresis. Standard Northern blot transfer to a nylon membrane (Hybond-N+2 Amersham Life Science, Little Chalfont, UK) was performed as described previously. The cDNA probes of p16, CDK4, and $\mathrm{Rb}$ were labeled by $\left[\alpha-{ }^{32} \mathrm{P}\right]$ deoxycytidine triphosphate using the random primer method (Prime-It II Kit; Stratagene, La Jolla, $\mathrm{CA}$ ). The membrane was hybridized with the probe in Rapid-hyb buffer (Amersham) according to the manufacturer's protocol. The membrane was exposed to a Kodak x-ray film (Eastman Kodak, Rochester, NY) between two intensifying screens at $-80^{\circ} \mathrm{C}$ for autoradiography. The $\beta$-actin cDNA probe was labeled as described above and used as an internal control for normalization.

\section{ELISA assay}

Cells were grown in 10-cm culture dishes and either untreated or transduced with control virus AdRSVlacZ or AdRSVp16 (at moi=200). After 90-min viral infection, viral medium was replaced with an exact $10 \mathrm{ml}$ fresh medium to each sample dish. The cell medium (supernatant) was collected $72 \mathrm{hr}$ after viral transduction, and cells attached on the culture dish were counted. The supernatant was processed to determine the secreted amount of $\mathrm{VEGF}_{165}$ protein by VEGF immunoassay kit (Quantikine VEGF ELISA Kit,
R\&D Systems, Minneapolis, MN). The procedures followed the methods according to the manufacturers' manual. The results were normalized based on the same amount of cells analyzed.

\section{$\mathbf{X}-\mathbf{G a l}$ staining for senescence-associated $\beta$-gal}

The activity of endogenous $B$-galactosidase, the biomarker for cells undergoing senescence, was used to detect any senescent cells. The cells were fixed in $1 \%$ glutaraldehyde, $10 \mathrm{mM} \mathrm{NaH}_{2} \mathrm{PO}_{4}, 150 \mathrm{mM} \mathrm{NaCl}$, and $1 \mathrm{mM} \mathrm{MgCl}_{2}$, at $37^{\circ} \mathrm{C}$ for 30 minutes. After a brief washing with PBS three times, the cells were then incubated at $37^{\circ} \mathrm{C}$ overnight in substrate solution containing $1 \mathrm{mg} / \mathrm{ml} \mathrm{X-Gal,} 5 \mathrm{mM}$ potassium ferricyanide, $5 \mathrm{mM}$ potassium ferrocyanide, $150 \mathrm{mM} \mathrm{NaCl}, 1$ $\mathrm{mM} \mathrm{MgCl}$, $40 \mathrm{mM}$ citric acid, and $40 \mathrm{mM} \mathrm{NaH}{ }_{2} \mathrm{PO}_{4}$ ( $\mathrm{pH}$ 6.0).

\section{In vivo animal model of a xenograft breast tumor growing in mice}

Mouse BCa JygMC(A) cells were either untreated, or transduced with AdRSVlacZ or AdRSVp16 at moi of 200. Forty-eight hours after viral infection, the cells were harvested and the viable cell numbers were counted in a hemocytometer using trypan blue exclusion. Cells $\left(1 \times 10^{7}\right.$ cells per mouse) were injected subcutaneously into the flanks of 8-week-old female nude mice (Harlan Sprague Dawley, Indianapolis, IN). Three groups of mice, with five mice in each group, were formed corresponding to the three groups of cells mentioned above. Mice were monitored every day. All the mice were sacrificed at day 28 post inoculation. The subcutaneous (s.c.) primary tumors were harvested, weighed, and processed to tumor sections for immunohistochemical (IHC) staining for proliferating cell nuclear antigen (PCNA) and CD31 expression.

\section{Immunohistochemistry staining of tumor sec- tions}

For tumor sections derived from JygMC(A) s.c. tumors grown on nude mice (see above), samples were processed for IHC staining as described previously (24). The tumor sections were first incubated with $1 \% \mathrm{H}_{2} \mathrm{O}_{2}$ for 30 minutes, then incubated with primary antibody either against PCNA (Santa Cruz Biotech, Santa Cruz, CA) or against CD31 (Pharmigen, BD Biosciences, San Diego, CA) for $16 \mathrm{~h}$ at $4^{\circ} \mathrm{C}$, and followed by corresponding secondary antibody and a Universal Elite avidin-biotin complex kit (Vector Laboratories, Burlingame, CA) according to the manufacturer's protocol. The reaction was visualized with DAB solution $\left(0.5 \mathrm{mg}\right.$ of $3,3^{\prime}$-diaminobenzidine in $0.01 \% \mathrm{H}_{2} \mathrm{O}_{2}$-PBS) for 1 to $4 \mathrm{~min}$. 
The quantitation of CD31 staining was represented as microvessel density, which was measured by the method as described before $(31,32)$. In brief, the areas of highest neovessel density (so called "hot spots") were identified by light microscopy after scanning the entire tumor section at low power. Then, individual microvessels were counted at high power (x200 field) in an adequate area (e.g., $0.74 \mathrm{~mm}^{2}$ per field using $x 20$ objective lens and x10 ocular). Any CD31-positive stained endothelial cells or clusters separated from adjacent vessels were counted as a single microvessel, even in the absence of vessel lumen. Five randomly selected "hot spots" field were counted from each tumor (at least 3 tumors/per mouse group) and the mean \pm SD were represented in the figure. Likewise, the PCNA-positive cells were quantitated by counting in 5 random fields and the mean and standard were presented.

\section{TUNEL assay}

For the terminal deoxynucleotidyl transferase-mediated dUTP nick end labeling (TUNEL) assay, the xenograft breast tumors (untreated control tumors, control virus or AdRSVp16 treated tumors) growing in nude mice were harvested at necropsy, fixed with freshly prepared $10 \%$ buffered neutral formalin (Fisher Scientific, Fair Lawn, NJ) overnight at room temperature, dehydrated in a gradual series of ethanol, and embedded in paraffin. Tissue sections were cut $4 \mathrm{~mm}$ thick, mounted on Superfrost Plus glass slides (Fisher, Scientific, Pittsburgh, PA), deparaffinized with xylenes, rehydraded in a gradual series of ethanol, washed in $\mathrm{H}_{2} \mathrm{O}$ and subjected to TUNEL staining by using the In Situ Cell Death Detection Kit (Boehringer Mannheim, Indianapolis, IN) according to the manufacture's instruction. The TUNEL-stained cells were counter-stained with propidium iodide (Sigma, St. Louis, MO) and visualized by fluorescence microscopy.

\section{Results}

\section{p 16 inhibits growth of $\mathrm{BCa}$ cells in vitro}

To determine the effects of ectopic p16 expression on $\mathrm{BCa}$ cell growth in vitro, human $\mathrm{BCa}$ MDA-MB-231 cells were transduced with AdRSVp16 at different moi for 5 days (dose-dependent assay) or at moi of 200 for various days (time-dependent assay). As a control for adenoviral transduction, AdRSV2C9, an adenoviral vector that expressed an unrelated gene was included as a control virus. The results showed that AdRSVp16 significantly inhibited the growth of MDA-MB-231 cells in both dose-dependent (Fig. 1A) and time-dependent (Fig. 1B) manner by comparing to the untreated control or control virus-transduced cells (Fig. 1).

To further examine whether p16 could inhibit other $\mathrm{BCa}$ cells in general, two more BCa cell lines, MCF-7 and JygMC(A), along with MDA-MB-231 were used for growth inhibition assay. The BCa cells were transduced with AdRSVp16 or control virus at moi=200, or no virus, respectively, and the cell numbers remained attaching on the culture dishes after five days were counted. We found that AdRSVp16 significantly inhibited the cell growth in all these BCa cells, with $49.2 \%, 59.1 \%$ and $40.5 \%$ inhibition of MDA-MB-231, MCF-7, and JygMC(A), respectively, compared to their untreated control counterparts (Fig. 2).

A
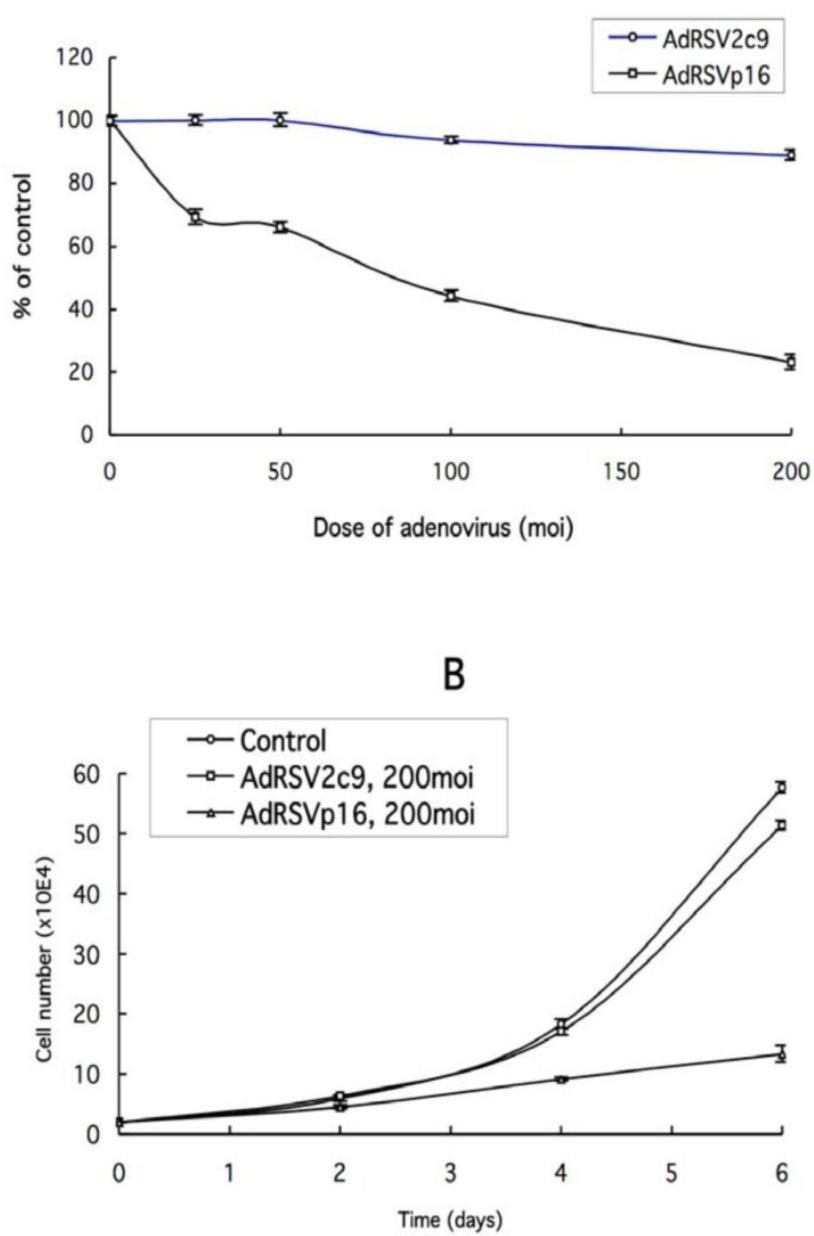

Figure I. Growth inhibitory effect of AdRSVpI6 on MDA-MB-23 I cells. (A) Dose-effect curve. Cells were plated in 6-well plates; after $24 \mathrm{~h}$ incubation, cells were either untreated or transduced with indicated moi control virus (AdRSV2C9) or AdRSVpl6. Cell numbers were counted using a Coulter cell counter at 5 days post-transduction. (B) Time-effect curve. Cells in 6-well plates were untransduced or transduced with moi of 200 control virus or AdRSVp 16. Cell numbers were counted using a Coulter cell counter at indicated days post-transduction. Data represent the results performed in duplicate. 


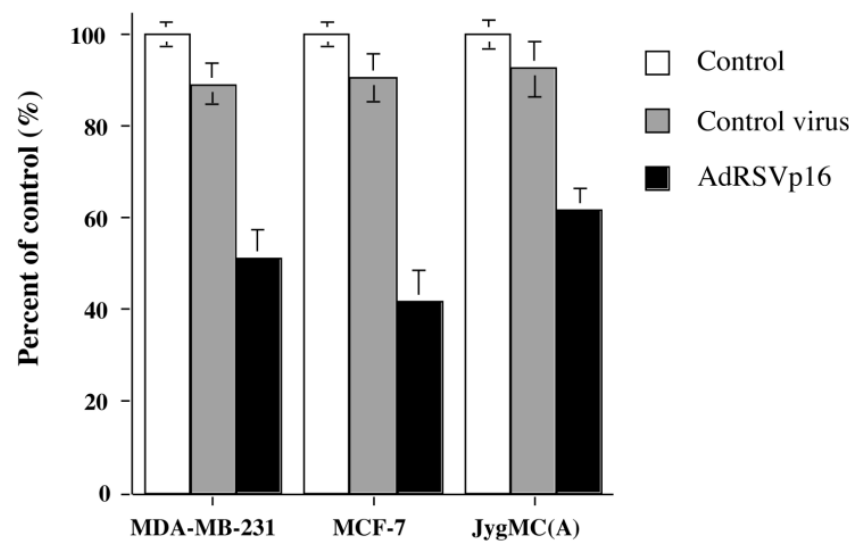

Figure 2. Effect of AdRSVpI 6 on growth of breast cancer cell lines. MDA-MB-23I, MCF-7 and JygMC(A) cells were untreated or transduced with either control virus or AdRSVpl6 $(m o i=200)$. Cell numbers were determined using a Coulter cell counter at day 6 after viral infection. The data represent results from two independent experiments, each performed in triplicate.

\section{p I6 inhibits BCa cell division}

To examine p16's effect on cell cycle distribution, a flow cytometry analysis was performed on synchronized MDA-MB-231 cells at different time after AdRSVp16 infection. In comparison to the untreated control or control virus treated cells, AdRSVp16-transduced MDA-MB-231 cells showed a significant accumulation of $\mathrm{G}_{0} / \mathrm{G}_{1}$ phase (cell-cycle-arrest cell population) and reduction of $S$ phase of cell population over time (Table 1). These results demonstrated a clear p16-mediated cell cycle arrest and inhibition of cell division. This apparent cell cycle arrest by p16 accounted primarily for the in vitro growth inhibition of MDA-MB-231 cells by AdRSVp16 (Fig. 2).

Table I. Cell population analysis by flow cytometry

\begin{tabular}{lllll}
\hline Days & Cells & $\mathrm{G}_{0} / \mathrm{G}_{1}(\%)$ & $\mathrm{S}(\%)$ & $\mathrm{G}_{2} / \mathrm{M}(\%)$ \\
\hline 2 & Untreated & 59.45 & 29.34 & 11.21 \\
& Control virus & 53.91 & 30.87 & 15.21 \\
4 & AdRSVp16 & 74.36 & 14.72 & 10.92 \\
& Untreated & 52.33 & 33.49 & 14.17 \\
& Control virus & 57.05 & 26.88 & 16.08 \\
& AdRSVp16 & 75.11 & 9.24 & 15.65 \\
\hline
\end{tabular}

MDA-MB-231 cells were synchronized by serum starvation in $0.5 \% \mathrm{FBS}$ at $37^{\circ} \mathrm{C}$ for $48 \mathrm{~h}$. The synchronized cells were replated in the medium containing $10 \% \mathrm{FBS}$ to reentering the cell cycle at $37^{\circ} \mathrm{C}$ for $16 \mathrm{~h}$, then the cells were transduced with control virus or AdRSVp16 at moi of 100. Cells were harvested at different time points post-transduction and analyzed for cell cycle distribution via propidium iodide staining as described in "Materials and Methods". The averages of the results from at least two independent experiments are presented.

\section{pl6 downregulates CDK4 expression}

p16 is a cyclin-dependent kinase (CDK4/6) inhibitor and a negative cell cycle regulator: by sequestering CDK4, p16 prevents the activation of CDK4/cyclin D, a complex which phosphorylates $\mathrm{Rb}$ protein to promote cell cycle progression (32). We were interested in investigating whether ectopic p16 expression in MDA-MB-231 cells would directly affect expression level of CDK4 and Rb, two key components responsible for the cell's orderly transition through the cell cycle. As shown in Fig. 3, ectopic p16 expression in MDA-MB-231 cells significantly reduced CDK4 mRNA expression while it had no detectable effect on $\mathrm{Rb}$ mRNA level. These results suggest that p16 may inhibit cell growth partially by directly reducing expression of CDK4 mRNA, in addition to p16's well-known inhibitory effect on activity of CDK4/ 6 by direct binding to them (46). Also as shown in the Fig. 3, the AdRSVp16-transduced MDA-MB-231 cells had an apparent induction of p16 mRNA as expected, confirming the functional gene transfer and expression of p16 by AdRSVp16.

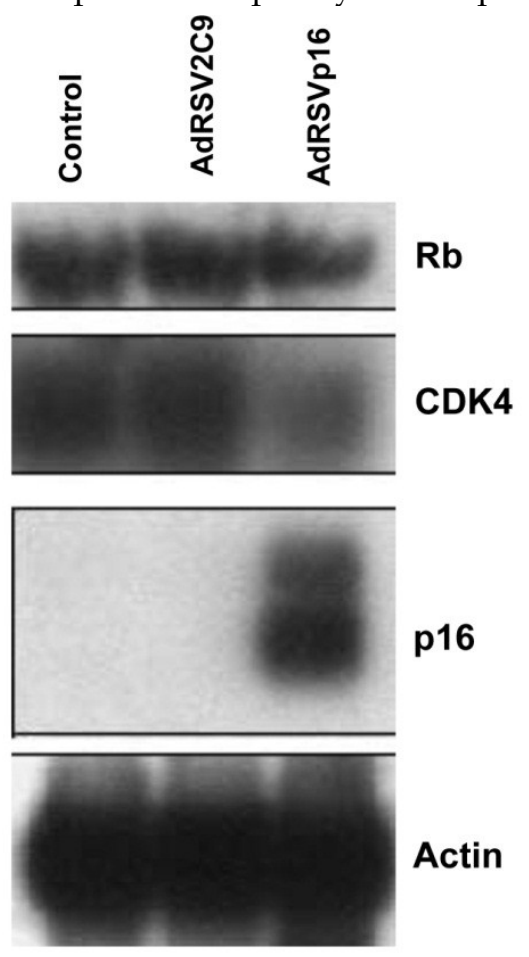

Figure 3. Northern blot analysis of pl6 mRNA in MDA-MB-23 I cells following AdRSVpI6 transduction. Cells were transduced with control virus or AdRSV 16 (moi=200) for $48 \mathrm{~h}$ and then the total RNA were isolated from the cells. Sample wells were each loaded with $10 \mu g$ of total RNA of each samples on a $12.5 \%$ agarose gel, electrophoresed and transferred to a nylon membrane, and hybridized with $32 \mathrm{P}$-labeled cDNA probes of Rb, CDK4, and pl6, respectively. The same blot was also hybridized with housekeeper gene $\beta$-actin 32P-labeled cDNA probe as an internal control. 


\section{p I 6 inhibits VEGF ligand secretion in MDA-MB-23 I cells}

As illustrated by our previous studies that ectopic p16 downregulates gene expression at the mRNA level of VEGF in MDA-MB-231 (17) and JygMC(A) cells (43), an angiogenic factor that plays pivotal role in the tumor angiogenesis, we intended to establish a cause-result relationship between p16's effect on VEGF production and angiogenesis. To determine whether p16 modulates the secretion of VEGF at the protein level, the active ligand form to the VEGF-mediated tumor angiogenesis, MDA-MB-231 cells were either untreated or transduced by control virus or AdRSVp16, and $72 \mathrm{~h}$ later the cell culture medium was collected to analyze the secreted form of VEGF protein by ELISA assay. The ELISA results showed that AdRSVp16-transduced MDA-MB-231 cells had significantly less VEGF protein $(66 \%$ inhibition) secreted into the medium compared to untreated control cells (Fig. 4).

\section{AdRSVpl 6 induced senescence in MDA-MB-23 I cells}

To determine whether the AdRSVp16 treated MDA-MB-231 cells were undergoing senescence, senescence-associated $\beta$-galactosidase at $\mathrm{pH} 6.0$ was measured by $\mathrm{X}$-gal staining. Whereas both untreated and control virus-transduced MDA-MB-231 cells had no detectable blue staining, AdRSVp16-transduced cells demonstrated the characteristic senescence-associated blue stained cytoplasm (Fig. 5C). The morphologic changes of AdRSVp16-transduced cells were also noticed: the AdRSVp16-transduced MDA-MB-231 cells were larger and flatter (Fig. 5C) than untreated (Fig. 5A) and control-virus treated cells (Fig. 5B).

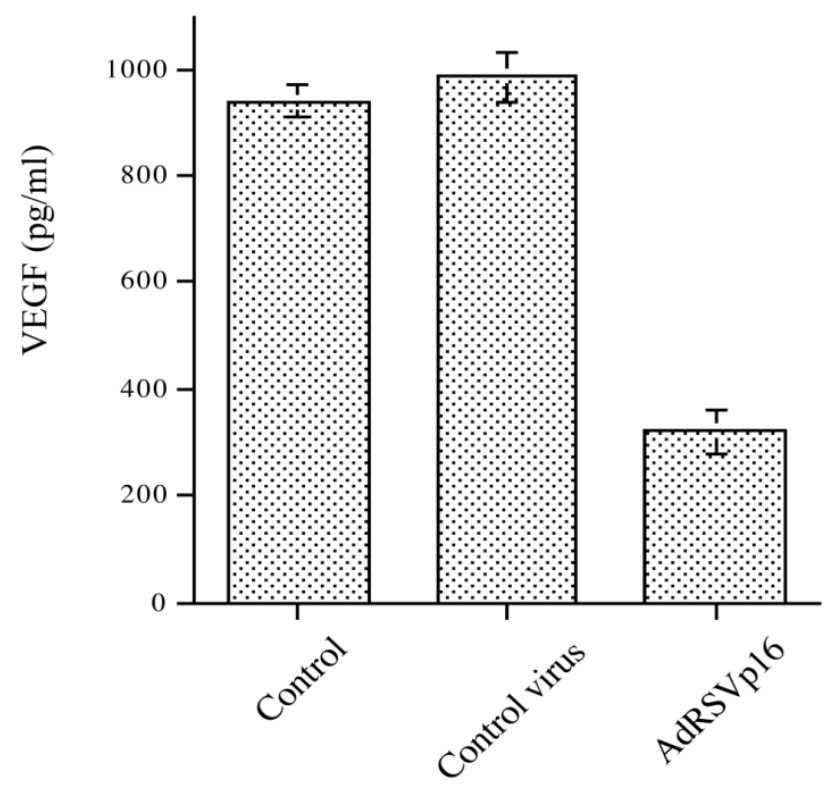

Figure 4. p I 6 decreased VEGF secretion by MDA-MB-23 I cells. Cells were grown in medium containing charcoal-stripped serum. Cells were either untreated or transduced with control virus AdRSVlacZ or AdRSVpl6 at moi of 200. The cell medium was collected $72 \mathrm{hrs}$ after viral transduction and subjected to VEGF determination by ELISA assay using a kit designated for human VEGFI65 (Quantikine VEGF ELISA Kit, R\&D Systems, Minneapolis, MN). The normalized results based on an equal amount of cells in each group are shown here.
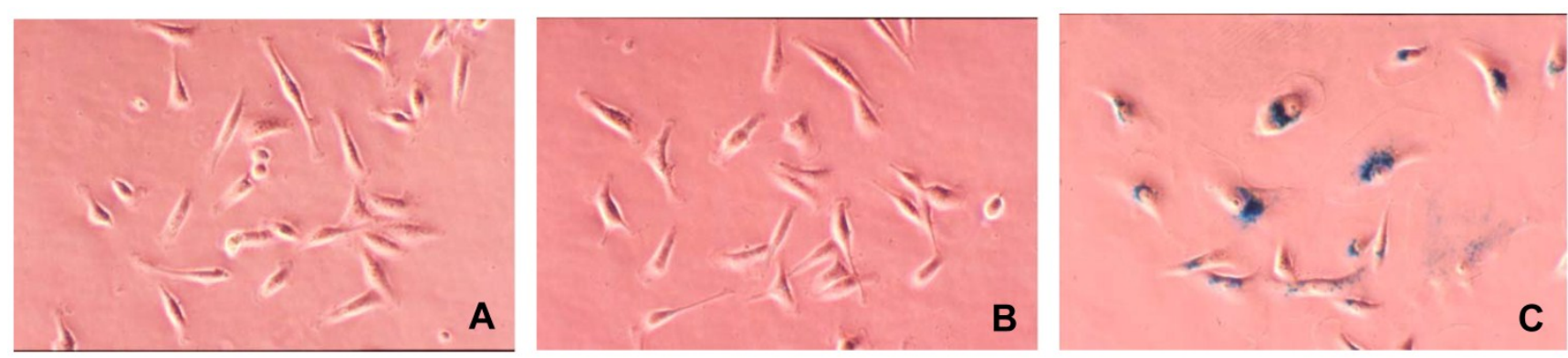

Figure 5. Induction of senescence by AdRSVpl6 in MDA-MB-23 I cells. Cells were untransduced (A) or transduced with 200 moi control virus AdRSV2C9 (B) or AdRSVpl6 (C). After incubation for 6 days post-infection, the senescence-associated $\beta$-gal was detected by $X-G a l$ staining (original magnification, $x 66$ ). 

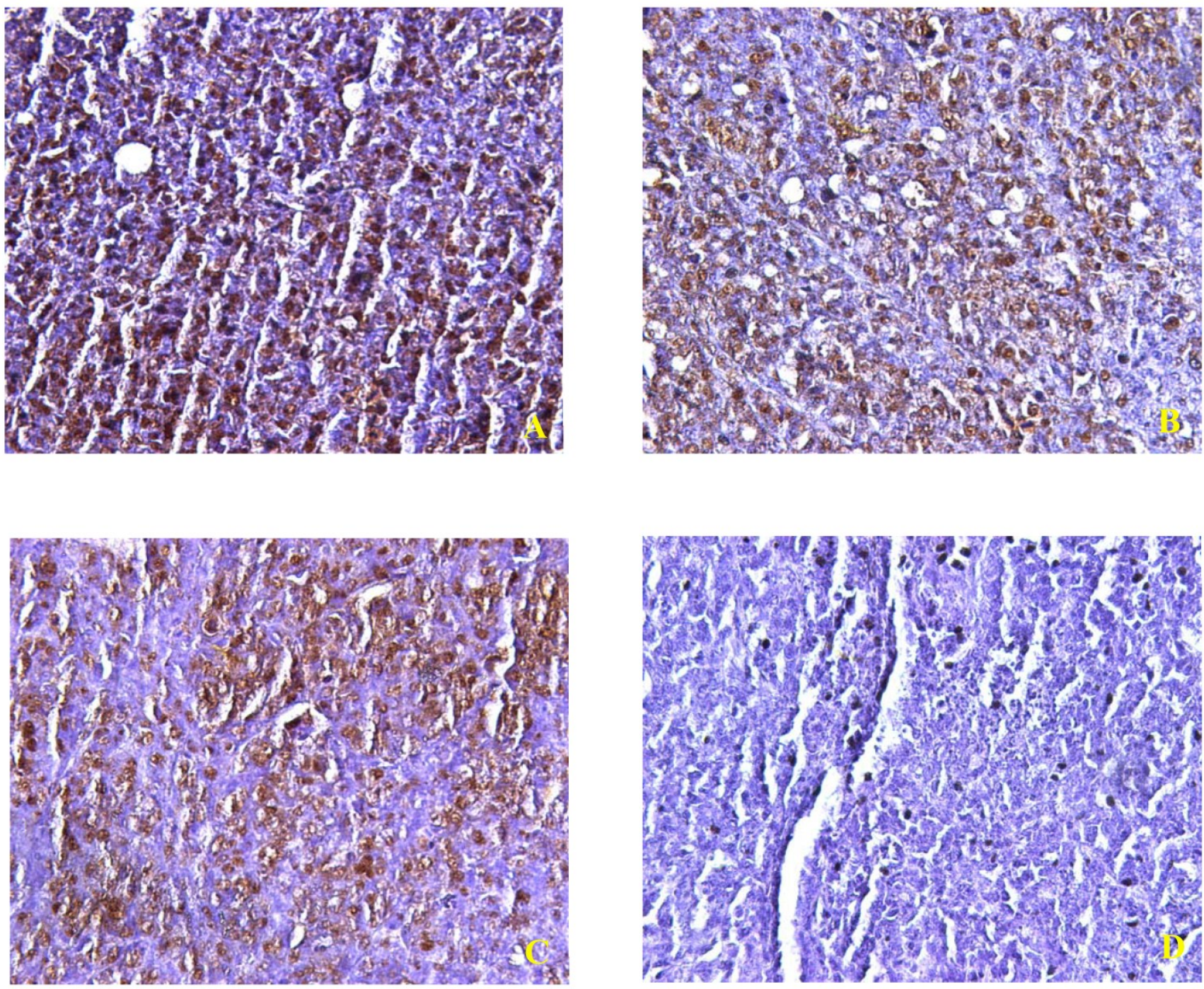

Figure 6. p 16 does not affect PCNA expression in breast tumors. Allograft breast tumors were established in nude mice by the subcutaneous injection of JygMC(A) cells, either untreated or had been transduced (moi=200 for $24 \mathrm{hr}$ ) with control virus or AdRSVpl6. The tumors were harvested 28 days after inoculation and tumor sections were subjected to immunohistochemical study using a primary antibody against PCNA. Shown are representative sections of PCNA staining for tumor sections from untreated control (A), control virus treated (B) and AdRSVpl6 treated (C) mice. D is a negative control in which the primary antibody was replaced by preimmune immunoglobulin G. The insert table is the quantitative presentation of above data by counting the PCNA positive cells in five randomly chosen fields in each group under the microscope. The average and the standard error are presented.

\section{p I 6 does not significantly affect proliferation inside breast tumor in vivo}

JygMC(A) breast tumors were established by s.c. injection of JygMC(A) cells that had been ex vivo transduced with either nothing, AdRSVlacZ or AdRSVp16 (ex vivo transduction at moi=200 for $48 \mathrm{~h}$ before injection), respectively. The tumors were harvested at necropsy and processed to tumor sections. The tumor sections were further subjected to IHC staining for proliferation marker PCNA inside the tumors (Fig. 6). PCNA expression was quantitated by counting the positive-stained cells in five randomly selected fields and the averages were presented and compared among the groups (Fig. 7). We found that p16 did not cause significant changes in the PCNA expression inside the tumors, implying that the suppression of the tumor mediated by p16 expression may not primarily result from p16's cell-cycle arrest function.

\section{pl 6 induces apoptosis both in vitro and in vivo}

Previous studies by others showed that p16 is able to induce cervical cancer cells to undergo apoptosis (19). As p16-mediated apoptosis may also serve as one of the means that contributes to p16's global 
inhibitory effect on BCa cell growth, we were interested in determining whether p16 causes any apoptosis in the BCa cells. To investigate whether p16 increases apoptotic population of the cells following AdRSVp16 transduction in vitro, AdRSVp16transduced MDA-MB-231 cells were analyzed by flow cytometry following propidium iodide staining. As shown in Table 2, the apoptotic population, as indicated by pre- $\mathrm{G}_{1}$ phase, was noticeably higher in AdRSVp16-transduced cells (12.9\%) than observed for untreated $(2.89 \%)$ and control virus transduced $(3.47 \%)$ counterparts. Likewise, TUNEL staining of the tumor sections harvested from AdRSVp16-treated tumors revealed a significant apoptosis (as indicated by bright yellowish fluorescence-stained cells) (Fig. 8B) compared to that of untreated tumors (Fig. 8A) and control virus treated tumors (not shown).

Table 2. Apoptotic cell population (pre- $\mathrm{G}_{1}$ ) analysis by flow cytometry

\begin{tabular}{lll}
\hline Cells & pre-G $\mathrm{G}_{1} /$ apoptosis $(\%)$ & $\begin{array}{l}\text { sum of remaining cell } \\
\text { population }\left(\mathrm{G}_{0} / \mathrm{G}_{1}, \mathrm{~S}\right. \\
\left.\text { and } \mathrm{G}_{2} / \mathrm{M}\right)(\%)\end{array}$ \\
\hline Untreated & 2.89 & 97.11 \\
Control virus & 3.47 & 99.53 \\
AdRSVp16 & 12.9 & 87.1 \\
\hline
\end{tabular}

MDA-MB-231 cells were either untreated or transduced with control virus (AdRSVlacZ) or AdRSVp16 at moi of 100. Both attached cells and cells in supernatant were collected at day 5 post viral transduction, stained with propidium iodide and processed for fluorescent flow cytometry as described in Materials and methods section. The percentages of cell population at pre- $\mathrm{G}_{1}$ (apoptosis) phase are presented. The averages of the results from at least two independent experiments are presented.

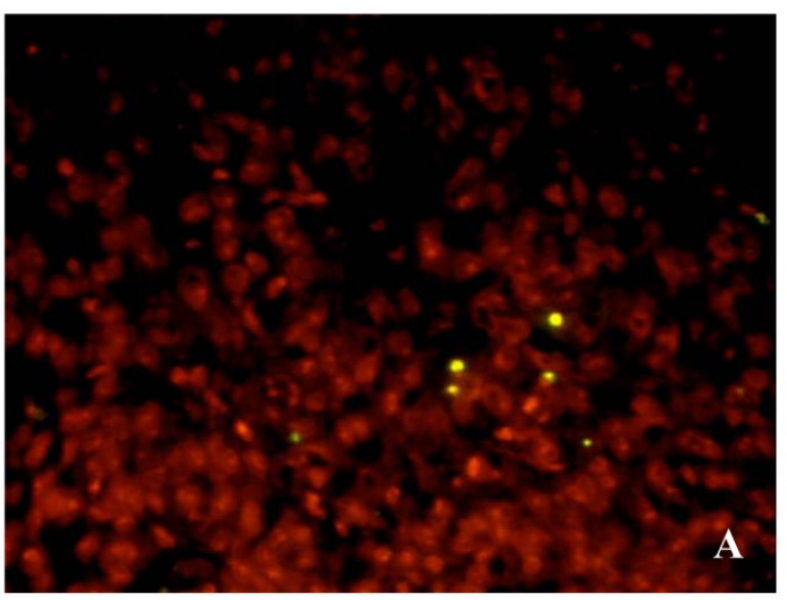

Control virus treated

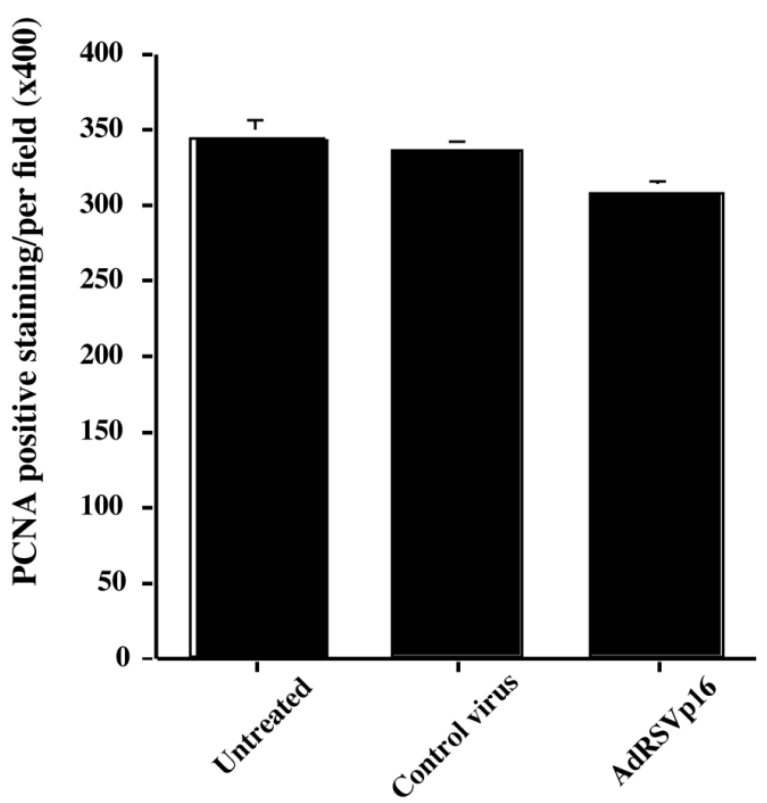

Figure 7. Quantitation of PCNA staining in breast tumors. The PCNA staining from Figure 6 is quantitized by counting positive PCNA-stained cells in five random fields. The mean and S.D. from each group are presented.

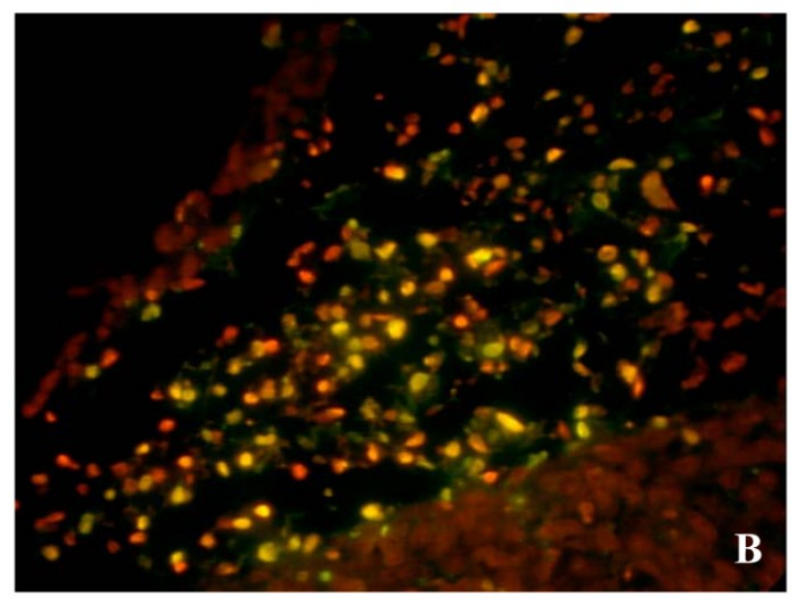

AdRSVp16 treated

Figure 8. p 16 induces apoptosis in breast tumors. Allograft breast tumors (see Fig. 6 legend) were collected and the tumor sections were subjected to TUNEL staining using the In situ Cell Death Detection kit. The sections were counterstained with propidium iodide (red). The apoptotic cells were illustrated by bright yellow color. Shown are the images of tumor section from untreated control $(A)$ and AdRSVp 6 treated (B) mice. Tumor section from control virus AdRSVlacZ-treated mice showed results similar to those in A (not shown). Original magnification, 40x. The apoptotic cells were counted in five random selected fields under a 20x magnification lens, the mean and S.D. of apoptotic cells in untreated control, AdRSVlacZ-treated and AdRSVpI6-treated tumor sections are 16 $\pm 2,17 \pm 3$, and I40 \pm 11 , respectively. The differences of apoptotic cells between AdRSVpl6-treated group and the other two groups are significant $(p<0.05)$. 

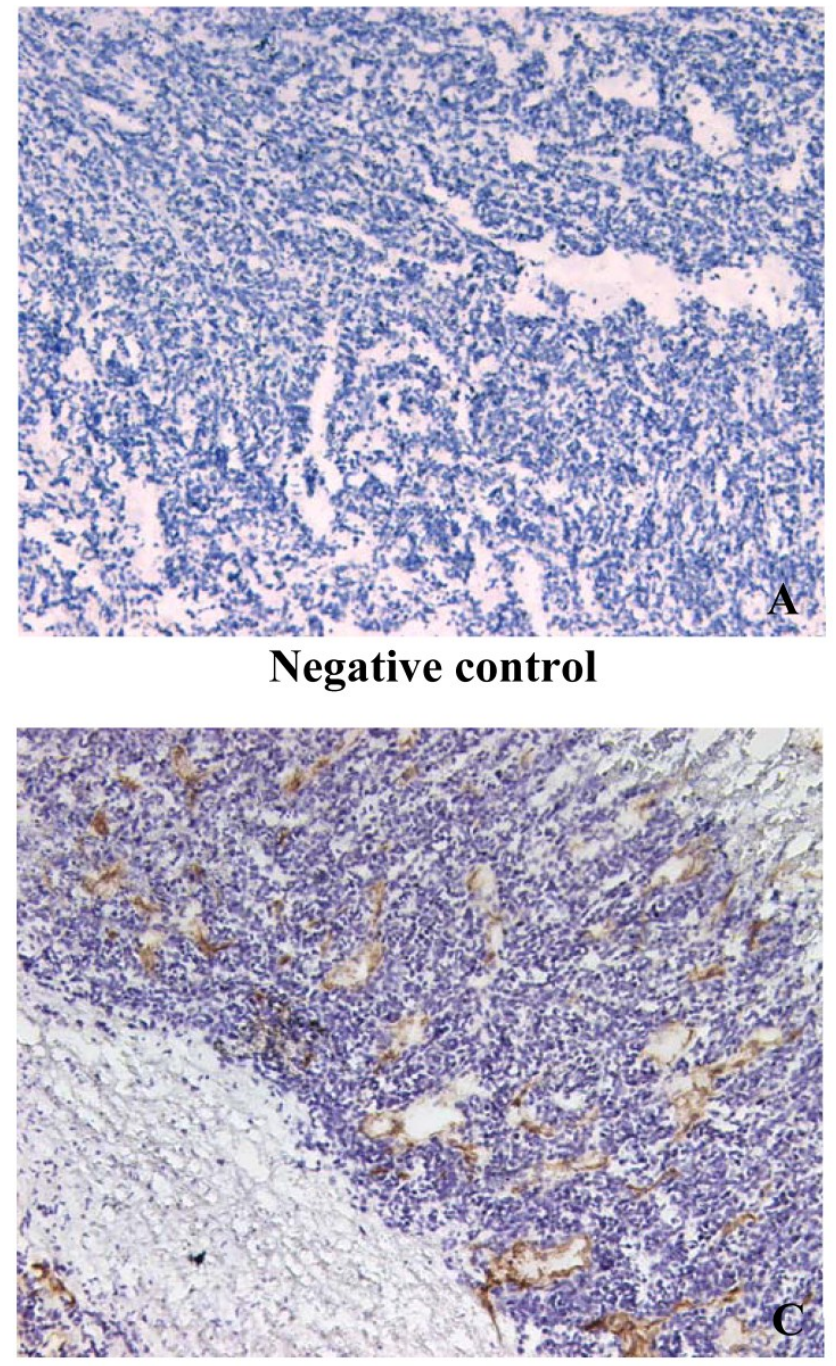

\section{Virus control}
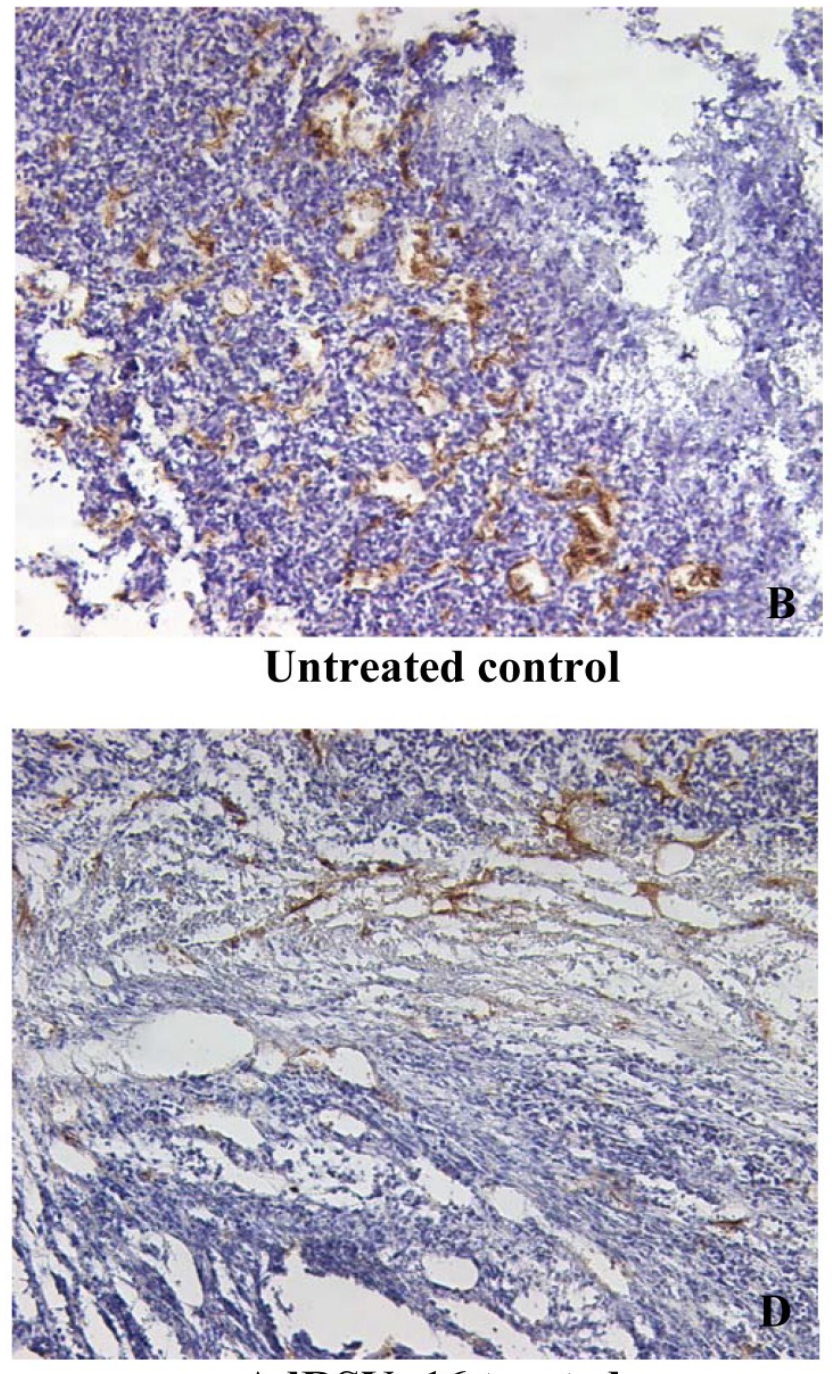

AdRSVp16 treated

Figure 9. pl6 inhibited angiogenesis in breast tumors. The s.c. allograft tumors were harvested at day 28 post tumor cell implantation. The tumor sections were analyzed by immunohistochemistry using anti-CD3 I primary antibody, followed by appropriate secondary antibody and procedures as described in Fig. I legend. Shown are CD3 I staining for tumor section from untreated control (B), control virus treated group (C), and AdRSVpl6-treated group (D). A is a negative control in which the primary antibody was replaced by preimmune immunoglobulin $\mathrm{G}$.

\section{p I 6 inhibits breast tumor angiogenesis}

To analyze p16's effects on anti-angiogenesis in vivo, the above-mentioned mouse tumor sections were subjected to IHC staining for neovascularization marker CD31 (Fig. 9). The AdRSVp16-treated tumor had much less CD31 expression (dark brown staining, Fig. 9D) compared to the untreated tumor (Fig. 9B) or control-virus treated tumor (Fig. 9C), suggesting that p16 expression inhibited tumor vascularization in vivo. These dark brown staining are specific for CD31 protein; because the negative control, in which the same IHC protocol on untreated tumor section was performed except for the omission of the use of the
anti-CD31 primary antibody, did not show any positive dark brown color (Fig. 9A). The quantitation of CD31 expression (presented as microvessel density, not shown) indicated that AdRSVp16-treated tumors had a $74 \%$ inhibition of angiogenesis compared to the untreated control whereas the control virus-treated tumors only had an insignificantly minor inhibition. These results suggest p16 expression inhibited microvessel formation, or angiogenesis, inside the tumors.

Examining the results of TUNEL assays and IHC staining of PCNA and CD31 from the tumor sections, we noticed that the difference between the AdRSVp16-treated tumors and the control vi- 
rus-treated tumors in CD31 staining (Fig. 6 \& Fig. 7) and TUNEL signal (bright yellow color, Fig. 8) appear much greater than that in PCNA signals (Fig. 6). These results indicate that p16 significantly inhibits angiogenesis (Fig. 9) and induces apoptosis (Fig. 8) in vivo while causes an insignificant change in PCNA expression (Fig. 7), implying p16 may have a greater effect on apoptosis/anti-angiogenesis than on anti-proliferation in vivo; namely, p16 may inhibit breast tumor in vivo growth primarily through anti-angiogenic effects.

\section{Discussion}

In summary, our studies have shown that adenoviral-mediated p16 expression inhibits BCa cell growth, blocks cell division, decreases VEGF secretion, induces senescence and apoptosis, and suppresses angiogenesis in BCa cells. To our knowledge, this study is the first report that simultaneously examines these multiple p16-mediated functions relevant to its global anti-tumor ability in one tumor cell system. These data together suggest that p16-mediated tumor suppression results from the combined effects of several p16's anti-tumor functions. Among them, some appear to contribute more than others; the effect of the same functions may vary from the in vitro to in vivo conditions.

Angiogenesis is an essential prerequisite for aggressive tumor proliferation and malignant progression as it provides nutrients and oxygen to the growing tumor $(34,35)$. This process (tumor angiogenesis) requires several angiogenic factors, primarily VEGF (36-38). The level of VEGF expression is correlated to the tumor-associated neovascularization and malignancy of the BCa specimens (12-16) while p16 expression inversely correlates with breast tumor malignancy. The downregulation of VEGF expression by ectopic p16, as shown by our previous studies (17), prompts the link of p16's ability on anti-angiogenesis. As confirmed by this study, p16 indeed suppresses tumor angiogenesis in terms of neovacularization; these results suggest that p16's anti-VEGF/angiogenesis effect may play an important role in p16's global effects on tumor suppression.

In our BCa model, ectopic p16 expression mediated by adenoviral vector significantly inhibited cell proliferation in vitro (Fig. 2), effectively blocking cell division and causing cell-cycle arrest at $\mathrm{G}_{0} / \mathrm{G}_{1}$ phase in BCa cells in vitro as indicated by cell cycle analysis (Table 1). Interestingly, we did not observe a significant difference in PCNA staining (marker for dividing cells or cell proliferation) between AdRSVp16-treated tumors and control tumors (Fig. 7) in our mouse model. In contrast, we observed a dramatic increase on TUNEL signals (indicating increased apoptosis) (Fig. 8) and significantly reduced CD31 staining (marker for neovascularization) (Fig. 9) in AdRSVp16-treated tumors compared to its controls. Based on the results from tumor sections in our mouse model, p16 appears to have a greater effect in vivo on anti-angiogenesis and induction of apoptosis than on anti-proliferation. In other words, these combined data imply that p16 may inhibit breast tumor in vivo growth primarily through anti-angiogenic effects.

The growth inhibition mediated by AdRSVp16 in our study is mainly from p16's effect, not from viral vector per se. Because we observed a similar growth inhibition in inducible p16 system in MDA-MB-231 cells: MDA-MB-231 cells stably expressing Tet-on p16 (MDA/Tet-on p16) had tightly regulated, inducible p16 expression under inducer doxycyclin (Dox) (45). By compared to MDA/Tet-on p16 cells without Dox, the cells with Dox (p16-expressing) had $45.6 \%$ inhibition on cell growth (45). While AdRSVp16-transduced MDA-MB-231 cells had a slightly higher inhibition of $49.25 \%$ compared to the untreated control (Fig. 2); the difference implies that adenoviral vector per se at moi=200 might cause some minor growth inhibition, as reflected by a minor inhibition of cell growth by the control vector (Fig. 2). However, the consistent results from MDA/Tet-on p16 cells have corroborated p16's anti-proliferation effect by AdRSVp16.

Numerous reports have shown that p16-mediated gene transfer can effectively suppress various cancer growth both in vitro and in vivo including in prostate cancer (39), glioma (40), cervical cancer (19), ovarian cancer (18), and colon cancer (19). Consistently, our previous studies have also indicated that adenoviral-mediated p16 gene transfer inhibited tumor growth of prostate cancer $(24,42)$ and BCa (17), and suppressed angiogenesis (17) and metastasis (43) in BCa. While the majority of these studies correlated the p16's anti-proliferation effect to the anti-tumor ability, the exploration of other p16's functions related to this matter, especially the anti-angiogenesis property, is under-investigated. Our studies showed that p16 inhibits BCa angiogenesis by suppressing VEGF expression $(17,44)$ and secretion (Fig. 4). p16 appears to downregulate VEGF gene expression at the transcriptional level (44) and our ongoing studies revealed that p16 directly binds to HIF- $1 \alpha$, the transcriptional regulator of the VEGF gene, and alters HIF-1 $\alpha$ 's cellular localization from the nucleus to the cytoplasm $(43,44)$. This finding suggests a potential mechanism responsible, at least in part, for p16's global anti-tumor effects is through p16-mediated anti-VEGF/angiogenesis function.

Moreover, our other recent studies revealed that 
p16 inhibits HIF-1 $\alpha$ /hypoxia-promoted cell migration in MDA-MB-231 cells (45). As cell migration plays an important role in the multiple-step process of metastasis, and hypoxia is the unique characteristic of tumor microenvironment, the anti-migration by $\mathrm{p} 16$ may reflect another potential p16's anti-tumor property. This is probably due to the observation that $\mathrm{p} 16$ can neutralize the effect of HIF-1 $\alpha$ (44), the transcriptional factor regulating cellular responses under hypoxia. Furthermore, our ongoing project also showed a preliminary result that MDA-MB-231 cells transduced with AdRSVp16 had a moderate reduction of Flk-1 (VEGF receptor 2) protein expression (not shown), suggesting that p16 may block the VEGF signaling pathway at two levels: one at the ligand level by downregulating VEGF; and the other probably at the receptor level by reducing Flk-1 expression.

$\mathrm{BCa}$ is the most frequently diagnosed cancer and the second leading cause of cancer deaths in American women today. It is estimated that there were 207,090 new cases and 39,840 deaths of BCa in the United States in 2010 (41). Research studies focusing on BCa treatment have increased dramatically in recent years. Some therapies appear to be effective with respect to the primary tumor. However, there are still no effective approaches to prevent and cure tumor metastasis, the ultimate cause of death for BCa patients. The relative success of controlling primary tumorigenesis has been confounded by a general failure to substantially reduce $\mathrm{BCa}$ deaths. Thus, a critical need exists to understand and develop effective treatments for those parameters contributing to $\mathrm{BCa}$ metastasis. As angiogenesis is an essential prerequisite for tumor metastasis, p16-mediated anti-VEGF/anti-angiogenesis should provide an effective mean to suppression of metastasis.

The lack of or decreased expression of tumor-suppressor-gene p16 is widely observed in many cancers including BCa $(25,30)$. In our study, all three $\mathrm{BCa}$ cell lines used in this study, MDA-MB-231, MCF-7 and JygMC(A), are p16 negative $(17,43)$ whereas MDA-MB-231 is estrogen receptor (ER)-negative and MCF-7 is ER-positive. These results indicate that restoring wild-type p16 in BCa cells can effectively inhibit BCa cells growth and tumor suppression regardless of the ER status of $\mathrm{BCa}$, suggesting that p16 gene transfer may have a wide clinical application for the treatment of $\mathrm{BCa}$ patients. In summary, with p16's combined effects on induction of senescence and apoptosis, inhibition on cell proliferation, VEGF expression and angiogenesis in BCa, p16 gene therapy may provide an effective therapy for $\mathrm{BCa}$ and prolong patients' survival when used in conjunction with other conventional therapeutic mo- dalities such as radiation and chemotherapy.

\section{Acknowledgements}

This research project was partially supported by NIH grants DK65962 (YL) and CA107162 (YL). We thank Andrew Lu for reviewing the manuscript.

\section{Competing Interests}

The authors have declared that no competing interest exists.

\section{References}

1. Shapiro GI, Rollins BJ. p16 $6^{\mathrm{INK} 4 \mathrm{~A}}$ as a human tumor suppressor. Biochem Biophys Acta 1996; 1242: 165-9.

2. Hussussian CJ, Struewing JP, Goldstein AM, et al. Germline p16 mutations in familial melanoma. Nat Genet 1994; 8: 15-21.

3. Caldas C, Hahn SA, De Costa LT, et al. Frequent somatic mutations and homozygous deletions of the p16 (MTS1) gene in pancreatic adenocarcinoma. Nat Genet 1994; 8: 27-32.

4. Cairns P, Polascik TJ, Eby Y, Sidransky D. High frequency of homozygous deletion at $\mathrm{p} 16 / \mathrm{CDKN} 2$ in primary human tumors. Nat Genet 1995; 11: 210-2

5. Jen J, Harper JW, Bigner SH, et al. Deletion of p16 and p15 genes in brain tumors. Cancer Res 1994; 54: 6353-8.

6. Hatta Y, Hirama T, Miller CW, et al. Homozygous deletions of the p15 (MTS2) and p16 (CDKN2/MTS1) genes in adult T-cell leukemia. Blood 1995; 85: 2699-704

7. Li YJ, Hoang-Xuan K, Delattre JY, et al. Frequent loss of heterozygosity on chromosome 9, and low incidence of mutations of cyclin-dependent kinase inhibitors p15 (MTS2) and p16 (MTS1) genes in gliomas. Oncogene 1995; 11: 597-600.

8. Xiao S, Li D, Corson JM, et al. Codeletion of p15 and p16 genes in primary non-small cell lung carcinoma. Cancer Res 1995; 55: 2968-71.

9. Chen Z-H, Zhang H, Savarese T. Gene deletion chemoselectivity; Codeletion of the gene for $\mathrm{p} 16^{\mathrm{INK}}$, methylthioadenosine phosphorylase, and the $\alpha$ - and $\beta$-interferons in human pancreatic cell carcinoma lines and its implications for chemotherapy. Cancer Res 1996; 56: 1083-90.

10. Dvorak HF, Nagy JA, Feng D, et al. Vascular permeability factor/vascular endothelial growth factor and the significance of microvascular hyperpermeability in angiogenesis. Curr Top Microbiol Immunol 1999; 237: 97-132.

11. Yoshiji H, Gomez DE, Shibuya M, Thorgeirsson UP. Expression of vascular endothelial growth factor, its receptor, and other angiogenic factors in human breast cancer. Cancer Res 1996; 56: 2013-6.

12. Obermair A, Kucera E, Mayerhofer K, et al. Vascular endothelial growth factor (VEGF) in human breast cancer: correlation with disease-free survival. Int J Cancer 1997; 74: 455-8.

13. Gasparini G, Toi M, Gion M, et al. Prognostic significance of vascular endothelial growth factor protein in node-negative breast carcinoma. J Natl Cancer Inst 1997; 89: 139-44.

14. Linderholm B, Tavelin B, Grankvist K, Henriksson R. Vascular endothelial growth factor is of high prognostic value in node-negative breast carcinoma. J Clin Oncol 1998; 16: 3121-8.

15. Heffelfinger SC, Miller MA, Yassin R, Gear R. Angiogenic growth factors in preinvasive breast disease. Clin Cancer Res 1999; 5: 2867-76.

16. Salven P, Perhoniemi V, Tykka H, et al. Serum VEGF levels in women with a benign breast tumor or breast cancer. Breast Cancer Res Treat 1999; 53: 161-6.

17. Lu Y, Zhang J, Beech DJ, et al. p16 downregulates VEGF and inhibits angiogenesis in breast cancer cells. Cancer Ther 2003; 1: 143-51.

18. Wolf JK, Kim TE, Fightmaster D, et al. Growth suppression of human ovarian cancer cell lines by the introduction of a p16 gene via a recombinant adenovirus. Gynecol Oncol 1999; 73: 27-34. 
19. Sandig V, Brand K, Herwig $S$, et al. Adenovirally transferred p16INK4/CDKN2 and p53 genes cooperate to induce apoptotic tumor cell death. Nat Med 1997; 3: 313-9.

20. Azuma H, Takahara S, Ichimaru N, et al. Marked prevention of tumor growth and metastasis by a novel immunosuppressive agent, FTY720, in mouse breast cancer models. Cancer Res 2002; 62: 1410-9.

21. Uhrbom L, Nister M, and Westermark B. Induction of senescence in human malignant glioma cells by p16 ${ }^{\text {INK4A }}$. Oncogene 1997; 15: 505-14.

22. Fueyo J, Gomez-Manzano C, Yung WK, et al. Adenovirus-mediated p16/CDKN2 gene transfer induces growth arrest and modifies the transformed phenotype of glioma cells. Oncogene 1996; 12: 103-10.

23. Steiner MS, Wang Y, Zhang Y, Lu Y. p16/MTS1/INK4A suppresses prostate cancer by both $\mathrm{pRb}$ dependent and independent pathways. Oncogene 2000; 19: 1297-306.

24. Steiner MS, Zhang Y, Farooq F, Lerner J, Wang Y, Lu Y. Adenoviral vector containing wild type p16 suppresses prostate cancer growth and prolongs survival by inducing cell senescence. Cancer Gene Ther 2000; 7: 360-72

25. Silva J, Silva JM, Dominguez G, et al. Concomitant expression of p16INK4a and p14ARF in primary breast cancer and analysis of inactivation mechanisms. J Pathol 2003; 199: 289-97.

26. Lu Y, Carraher J, Zhang Y, et al. Delivery of adenoviral vectors to the prostate for gene therapy. Cancer Gene Ther 1999; 6: 64-72.

27. Graham FL, Prevec L. Gene transfer and expression protocols. In: Murray EJ, ed. Methods in Molecular Biology. New Jersey: Humana Press; 1991: 109-128.

28. Park M, Koff A. In: Current Protocols in Cell Biology; Bonifacino JS, et al, eds; 8.3.1-8.3.16. New Jersey: John Wiley \& Sons, Inc. 1998.

29. Zhang XW, Qing C, Xu B. Apoptosis induction and cell cycle perturbation in human hepatoma hep G2 cells by 10-hydroxycamptothecin. Anticancer Drugs 1999; 10: 569-76.

30. Brenner AJ, Paladugu A, Wang H, et al. Preferential loss of expression of p16(INK4a) rather than p19(ARF) in breast cancer. Clin Cancer Res 1996; 2: 1993-8.

31. Weidner N, Semple JP, Welch WR, Folkman J. Tumor angiogenesis and metastasis--correlation in invasive breast carcinoma. N Engl J Med 1991; 324: 1-8.

32. Hiraga T, Kizaka-Kondoh S, Hirota $K$, et al. Hypoxia and hypoxia-inducible factor-1 expression enhance osteolytic bone metastases of breast cancer. Cancer Res 2007; 67: 4157-63.

33. Itoh $\mathrm{N}$, Kakehi $\mathrm{Y}$, Akao $\mathrm{T}$, et al. Concomitant presence of p16/cyclin-dependent kinase 4 and cyclin D/cyclin-dependent kinase 4 complexes in LNCaP prostatic cancer cell line. Jpn J Cancer Res 1997; 88: 229-33.

34. Takeda A, Stoeltzing O, Ahmad SA, et al. Role of angiogenesis in the development and growth of liver metastasis. Ann Surg Oncol 2002; 9: 610-6.

35. Folkman J. Tumor angiogenesis: therapeutic implications. N Engl J Med 1971; 285: 1182-6.

36. Brem SS, Gullino PM, Medina D. Angiogenesis: a marker for neoplastic transformation of mammary papillary hyperplasia. Science 1997; 195: 880-1.

37. Brem SS, Jensen HM, Gullino PM. Angiogenesis as a marker of preneoplastic lesions of the human breast. Cancer 1978; 41: 239-44.

38. Jensen HM, Chen I, DeValut MR, and Lewis AE. Angiogenesis induced by "normal" human breast tissues; a probable marker for precancer. Science 1982; 218: 293-5.

39. Gotoh A, Kao C, Ko S-C, et al. Cytotoxic effects of recombinant adenovirus p53 and cell cycle regulator genes (p21 WAF1/CIP1 and p16CDKN4) in human prostate cancers. J Urol 1997; 158: 636-41.

40. Arap W, Nishikawa R, Furnari FB, et al. Replacement of the pl6/CDKN2 gene suppresses human glioma cell growth. Cancer Res 1995; 55: 1351-4.

41. Jemal A, Siegal R, Xu J, Ward E. Cancer Statistics 2010. CA: A Cancer J Clin 2010; 60: 277-300.

42. Allay JA, Steiner MS, Zhang Y, Reed CP, Crockroft J, Lu Y. Adenovirus p16 gene therapy for prostate cancer. World J Urol 2000; 18: 111-20.

43. Zhang J, Lu A, Beech D, Jiang B, Lu Y. Suppression of breast cancer metastasis through the inhibition of VEGF-mediated tumor angiogenesis. Cancer Ther 2007; 5: 273-86.
44. Zhang J, Lu A, Li L, Yue J, Lu Y. p16 modulates VEGF expression via its interaction with HIF-1 $\alpha$ in breast cancer cells. Cancer Invest 2010; 28: 588-97.

45. Li L, Lu Y. Inhibition of hypoxia-induced cell motility by p16 in MDA-MB-231 breast cancer cells. J Cancer 2010; 1: 126-135.

46. Ohtani N, Mann DJ, Hara E. Cellular senescence: its role in tumor suppression and aging. Cancer Sci 2009; 100: 792-7. 